\title{
Fluorescence Imaging of Rotational and Vibrational Temperature in a Shock Tunnel Nozzle Flow
}

\author{
Philip C. Palma* Paul M. Danehy ${ }^{\dagger}$ and A. F. P. Houwing ${ }^{\ddagger}$ \\ The Australian National University, Canberra, 0200, Australia
}

\begin{abstract}
Two-dimensional rotational and vibrational temperature measurements were made at the nozzle exit of a free-piston shock tunnel using Planar Laser-Induced Fluorescence. The Mach 7 flow consisted predominantly of nitrogen with a trace quantity of nitric oxide. Nitric oxide was employed as the probe species and was excited at $225 \mathrm{~nm}$. Non-uniformities in the distribution of nitric oxide in the test gas were observed, and concluded to be due to contamination of the test gas by driver gas or cold test gas. The nozzle-exit rotational temperature was measured and is in reasonable agreement with test gas. The nozzle-exit rotational temperature was measured and is in reasonable agreement with
computational modelling. Non-linearities in the detection system were responsible for systematic errors in the measurements. The vibrational temperature was measured to be constant with distance from the nozzle exit, indicating it had frozen during the nozzle expansion.
\end{abstract}

\section{Introduction}

C RUCIAL to the design of future aerospace vehicles is the accurate prediction of physical and chemical effects influencing the aerodynamics at hypersonic velocities. Realistic evaluation of hypersonic phenomena on a particular vehicle design can be managed in one of three ways: flight tests of actual vehicles; simulation of flight conditions on scale models in ground-based test facilities; or numerical simulations implementing models of physical and chemical processes - known as computational fluid dynamics (CFD). The first case is prohibitively expensive and reserved for the prototype design. Individual ground-based facilities can only simulate flow conditions for a portion of the flight trajectory. The flows produced by such facilities only partially simulate flight flow conditions. The latter method, CFD, is a promising alternative with the advent of low-cost, highpower computing resources. However, CFD codes require validation against experimental data before they can be confidently used to predict inflight behaviour and aid in vehicle design and performance evaluation. The experimental data must come from the first two methods mentioned above, therefore emphasising the close relationship that exists between the alternative approaches.

The shock tunnel is a ground-based test facility capable of producing very high enthalpies and shock-layer temperatures. It consists of a converging-diverging nozzle attached to the end of a shock tube. The performance of the shock tunnel can be enhanced by employing the free-piston driver technique, ${ }^{1}$ thus making it capable of producing chemically reacting flow phenomena (real-gas effects). Shock-tunnel flows generally only last for a few milliseconds due to the impulse nature of the shock tube, and as necessitated by the extreme gas temperatures attained.

Many optical techniques have been used for flow visualisation in shock tunnels and to complement surface pressure and heat-transfer measurements. Schlieren photography, shadowgraph and interferometry have been used extensively to provide two-dimensional measurements with high temporal resolution. However, they can only provide an integrated

*Postdoctoral Research Associate. Current address: School of Mechanical, Materials, Manufacturing Engineering and Management, Universily of Nollingham, Nolingham, NG72RD, England. AIAA Member.

${ }^{\dagger}$ Research Scientist. Current address: Mail Stop 236, NASA Langley Research Center, Hamplon VA 23681-2199, USA. AIAA member.

\$Senior Lecturer, Department of Physics, The Australian National University, Canberra, 0200, Australia. AIAA member.

Presented as Paper 98-2703 at the AIAA 20th AIAA Advanced Measurement and Ground Testing Technology Conference, Albuquerque, NM, Jun. 15-18, 1998; received Jun. 15, 2001; revision received Jul. 25, 2001; accepted for publication Sep. 18,2001 . Copyright (C) 2001 by the American Institute of Aeronautics and Astronautics, Inc. No copyright is asserted in the United States under Title 17, U.S. Code. The U.S. Government has a for Governmental Purposes. All other rights are reserved by the copyright owner. measurement along the line-of-sight through the flow which masks any three-dimensional behaviour in the flow. Planar laser-induced fluorescence (PLIF) has been widely used for flow visualisation in subsonic and supersonic flows and as a combustion diagnostic. ${ }^{2,3}$ It provides high spatial and temporal resolution, two-dimensional quantitative measurements and is chemical species specific. PLIF uses a thin sheet of laser light to excite a radiative transition in a particular chemical species in the flow. The laser sheet interrogates a thin $(\leq 1 \mathrm{~mm})$ cross section through the flowfield, allowing PLIF to resolve three-dimensional flow features. The short duration of the laser pulse and resulting fluorescence makes PLIF particularly useful for impulse facilities where the flow may only last for a millisecond.

The high-enthalpy flows produced by free-piston shock tunnels have a unique set of experimental problems not encountered in other flow facilities. Flows generated by free-piston shock tunnels have large pressure and temperature variations which cause large fluorescence signal variations due to high collisional quenching rates and changes to the spectral overlap integral between the laser and the absorption transition. ${ }^{4,5}$ Other problems include excessive laser-beam absorption which can lead to hole burning of the laser spectral profile, ${ }^{6}$ spectral interference from other flow species and fluorescence trapping. A more severe problem is that metallic contaminants which are eroded from the shock tube walls produce strong emission in high-temperature regions in the flowfield. ${ }^{7}$ This background emission can easily overwhelm the fluorescence signals, so careful spectral and temporal filtering of the signal is necessary. This remains the greatest obstacle for the application of PLIF to shock tunnels.

In order to validate complex flowfields calculated using CFD codes with experimental data from ground-based facilities, one must have an accurate knowledge of the freestream conditions produced by the facility. Measurements of rotational and vibrational temperatures, static pressure, velocity, and chemical species number densities are necessary to completely specify the thermodynamic state of the freestream. In the past, CFD codes have been used to predict these freestream parameters based on calibrations with pitot-pressure data, but little work has been performed on testing the validity of these nozzle-flow calculations. In the current study, we attempt to address this research shortfall by concentrating our efforts on measuring rotational and vibrational temperatures for nitric oxide (NO) using PLIF.

The process of validating a CFD code can be a particularly arduous one. The methodology and strategies used are therefore very important. The process of validation should commence with the study of simple flows (i.e., continuum, no real-gas effects) and gradually increase complexity of the flow (i.e., increase Mach number, add chemical effects). 
Experiments should be designed to test a single flow phenomenon while minimizing competing effects that may complicate data interpretation (cause-and-effect tests). Simple models can be used to test specific flow phenomena and in some cases this is more desirable. Following this methodology, the flow employed in the experiments presented here was a low-enthalpy nitrogen flow. The effects of chemistry are removed, simplifying the problem considerably. The vibrational temperature freezes during the nozzle expansion, and the temperature at which this freezing occurs has a significant influence on the nozzle-exit rotational temperature.

For example, consider the current flow conditions calculated with a quasi-one-dimensional inviscid nozzle-flow code (described later in this article) where the vibrationalfreezing temperature and chemistry can be varied. If we calculate the two extreme cases for vibrational freezing, one where the vibrational temperature is held in equilibrium with the translational temperature throughout the nozzle expansion and the other where it is frozen at the nozzlereservoir temperature, we get $560 \mathrm{~K}$ and $345 \mathrm{~K}$, respectively, for the nozzle-exit rotational temperature. By comparison, the effect of switching chemistry between equilibrium and non-equilibrium produces only a $20-\mathrm{K}$ difference at these operating conditions. Hence, by measuring the rotational temperature one can get a measure of how much energy is stored in the vibrational modes of the test gas, and therefore infer the vibrational-freezing temperature of $\mathrm{N}_{2}$.

Ideally one would like to measure the vibrational temperature, in this case of $\mathrm{N}_{2}$ since it is the predominant test gas species (for example, using broadband CARS ${ }^{8}$ ). Unfortunately in the current work we can only measure the NO vibrational temperature with PLIF. Since NO is a minority species in the flow (1\%) its vibrational-freezing temperature has a negligible effect on the nozzle-exit conditions. It can, however, be used to test the NO vibrational-energy transfer rates used in the nozzle-flow code.

In order to use NO PLIF to measure temperature, a small amount of NO must be present in the flow. Nitric oxide is stable and can be easily seeded into the test gas, ${ }^{9,10}$ however, this is not necessary here because of the high temperature $(4430 \mathrm{~K})$ achieved in the nozzle-reservoir region. A small amount of $\mathrm{O}_{2}$ added to the test gas is sufficient to produce NO at the nozzle exit without the need for handling toxic NO. The $\mathrm{O}_{2}$ dissociates in the nozzle-reservoir region and recombines with nitrogen atoms to form $\sim 1 \%$ NO. This value is constant downstream of the nozzle throat as the flow becomes chemically frozen. This approach also provides an opportunity to test the PLIF technique without complicating effects from other flow species (e.g., $\mathrm{O}_{2}$ ), or from excessive beam attenuation caused by high NO concentrations which are typical of air flows.

A recent PLIF study ${ }^{11}$ on an arc-heated facility at similar flow conditions to the current experiment produced good results, however, due to the running time of several hours, the experimenters were afforded the luxury of scanning their laser across many excitation lines to build up a spectrum. They observed vibrational nonequilibrium which was also confirmed by $\mathrm{N}_{2}$-CARS experiments. ${ }^{12}$ Very little work has been performed on impulse facilities due to the difficulties it entails. The objective of the current work is to explore these difficulties and develop PLIF to the stage where it can be used alongside other diagnostics regularly employed on shock tunnels.

\section{PLIF Theoretical Considerations}

\section{Laser-Induced Fluorescence Theory}

The theory of PLIF is well developed ${ }^{2,3}$ and only the points relevant to the current work will be discussed here. In particular, we will present a general theory and then make approximations which are relevant for the current application.

In a typical PLIF experiment, a laser is tuned to an optically-allowed electronic resonance of a particular molec- ular species. The molecules in a particular electronicrotational-vibrational level $m v^{\prime \prime} J^{\prime \prime}$ (typically the ground state) are excited to an electronic-rotational-vibrational level $n v^{\prime} J^{\prime}$. Molecules in the excited level $n v^{\prime} J^{\prime}$ can then emit radiation (fluorescence) which is collected by a detector (typically a two-dimensional CCD array). Molecules may also undergo collisions with other molecules or atoms which cause a transfer of population out of the laser coupled state $n v^{\prime} J^{\prime}$ to nearby rotational levels. This is known as rotational-energy transfer (RET). These collisionallyexcited nearby rotational levels also fluoresce, resulting in a broadband fluorescence signal. Similarly, there may also be vibrational-energy transfer (VET) from the excited vibrational level. Other collisions may cause some of the molecules in the excited state to be de-excited nonradiatively (collisional quenching) which produces a corresponding decrease in the total amount of fluorescence signal.

The number of fluorescence photons reaching a single pixel of the CCD detector is given by ${ }^{3}$

$$
N_{\mathrm{p}}=N_{\mathrm{T}} f_{\mathrm{B}} B_{J^{\prime} J^{\prime \prime}} E G \Phi \frac{\Omega}{4 \pi} \eta \ell
$$

where $N_{\mathrm{T}}\left[\mathrm{cm}^{-3}\right]$ is the number density of absorbers; $f_{\mathrm{B}}$ is the Boltzmann fraction; $B_{J^{\prime} J^{\prime \prime}}\left[\mathrm{s}^{-1}\left(\mathrm{~W} / \mathrm{cm}^{2} / \mathrm{cm}^{-1}\right)^{-1}\right]$ is the Einstein absorption coefficient for this particular rotationalvibrational transition; $E[\mathrm{~J}]$ is the energy of the laser pulse; $G\left[1 / \mathrm{cm}^{-1}\right]$ is the spectral overlap integral; $\Phi$ is the fluorescence yield; $\Omega$ is the solid angle subtended by the detector; $\eta$ is the detection efficiency; and $\ell[\mathrm{cm}]$ is the length of the interaction volume in the direction of the beam. The interaction volume is defined as that volume in the flow whose fluorescence is collected by a single detector pixel. The length and breadth of the volume are determined by the dimensions of the pixel, and the depth is determined by the thickness of the laser sheet.

Each of the parameters in Eq. (1) shall now be defined in more detail. The number density of absorbers can be defined by $N_{\mathrm{T}}=(p \chi / k T)$ where $p[\mathrm{~Pa}]$ is pressure, $\chi$ is NO mole fraction, $T[\mathrm{~K}]$ is the kinetic temperature and $k$ is the Boltzmann constant. The Boltzmann fraction is given by

$$
f_{\mathrm{B}}=\frac{2 J^{\prime \prime}+1}{Z_{\text {total }}} \exp \left(\frac{-F_{J^{\prime \prime}}}{k T_{\text {rot }}}\right) \exp \left(\frac{-G_{v^{\prime \prime}}}{k T_{\text {vib }}}\right) .
$$

Here, we have indicated the rotational and vibrational contributions to $f_{\mathrm{B}}$ and made the distinction between the rotational temperature $T_{\text {rot }}$ and vibrational temperature $T_{\text {vib }}$. $F_{J^{\prime \prime}}$ is the rotationally dependent part of the energy of the absorbing state and $G_{v^{\prime \prime}}$ is the vibrational energy. $Z_{\text {total }}$ is the total partition function.

The fluorescence yield $\Phi$ represents the ratio of deexcitation transitions that produce fluorescence photons to the total number of de-excitation transitions. It is given by

$$
\Phi=\frac{A_{\text {eff }}}{A_{\text {total }}+Q},
$$

where $A_{\text {eff }}\left[\mathrm{s}^{-1}\right]$ is the effective spontaneous emission rate for the collected fluorescence, $A_{\text {total }}\left[\mathrm{s}^{-1}\right]$ is the sum of spontaneous emission rates for all possible radiative transitions from the excited level, and $Q\left[\mathrm{~s}^{-1}\right]$ is the collisional quenching rate. $A_{\text {eff }}$ represents the way that $A_{\text {total }}$ is modified by spectrally selective elements in the detection chain (e.g., filters, camera quantum efficiency). Therefore the effective emission rate is given by ${ }^{13}$

$$
A_{\text {eff }}=\sum_{v^{\prime \prime}} T_{\lambda v^{\prime \prime}} A_{v^{\prime} v^{\prime \prime}}
$$

where $T_{\lambda v^{\prime \prime}}$ is the spectral transmission function for the detection system at the wavelength $\lambda$ and the summation is over all vibrational bands of the lower electronic state. 
The spectral-overlap integral $G\left[1 / \mathrm{cm}^{-1}\right]$ represents the degree of overlap between the irradiance lineshape $L(\nu)$ $\left[1 / \mathrm{cm}^{-1}\right]$ and the absorption lineshape $Y(\nu)\left[1 / \mathrm{cm}^{-1}\right]$. It is given by

$$
G=\int Y(\nu) L(\nu) d \nu
$$

where $Y(\nu)$ and $L(\nu)$ are both normalised such that $\int L(\nu) d \nu=1$ and $\int Y(\nu) d \nu=1$. The absorption lineshape defined above is derived from the definition of the spectral absorption coefficient, which is given by

$$
k_{\nu}=S_{12} Y(\nu),
$$

where $S_{12}\left[\mathrm{~cm}^{-2}\right]$ is the line-strength factor for the transition. With $k_{\nu}$ expressed in this manner, $S_{12}$ contains only terms relating to the transition probability and is independent of lineshape effects. The exact form of the lineshape function is determined by various spectral-line broadening processes.

\section{Thermometry}

There are many different approaches to LIF thermometry, ${ }^{2,3,14}$ but here we discuss only the method used in the current experiments. This method is based on the two-line planar-thermometry technique of McMillin ${ }^{9}$ and Palmer. ${ }^{10}$ Consider the temperature and rotational-level dependence of each quantity in Eq. (1). Hence, we have

$$
N_{\mathrm{p}} \propto E N_{\mathrm{T}}(T) f_{\mathrm{B}}(T, J) B(J) G(T, J) \Phi(T, J),
$$

where, for simplicity $B=B_{J^{\prime} J^{\prime \prime}}, T=T_{\text {rot }}$ and $J=J^{\prime \prime}$. Now, if we make fluorescence measurements by exciting two different rotational levels (denoted by numbers 1 and 2), and take the ratio of the signals, we obtain

$$
\text { Signal Ratio }=\frac{N_{\mathrm{p} 1}}{N_{\mathrm{p} 2}}=\mathcal{C} \frac{E_{1}}{E_{2}} \frac{B_{1}}{B_{2}} \frac{f_{\mathrm{B} 1}}{f_{\mathrm{B} 2}},
$$

where $\mathcal{C}$ is a constant and we have assumed the quantities $G(T, J)$ and $\Phi(T, J)$ are independent of the excitation level $J$ and hence any temperature dependence cancels out in the ratio. Also, we have assumed that the number density of NO molecules $N_{\mathrm{T}}(T)$ remains constant between the two measurements and also cancels. We will justify these assumptions below. The laser energy $E$ is not dependent on $J$ but usually fluctuates from pulse-to-pulse and so is retained for completeness. Equation (8) shows that the only temperature dependence is through the Boltzmann fraction, and this is the basis of most LIF thermometry techniques. Substituting for $f_{\mathrm{B}}$ from Eq. (2) and assuming that each transition is within the same vibrational band (i.e., $G_{v^{\prime \prime}}$ is constant), we obtain

$$
\frac{N_{\mathrm{p} 1}}{N_{\mathrm{p} 2}}=\mathcal{C} \frac{E_{1} B_{1}\left(2 J_{1}+1\right)}{E_{2} B_{2}\left(2 J_{2}+1\right)} \exp \left(\frac{-\left(F_{J 1}-F_{J 2}\right)}{k T_{\text {rot }}}\right),
$$

and, solving for $T_{\text {rot }}$,

$$
T_{\text {rot }}=\frac{\left(F_{J 2}-F_{J 1}\right) / k}{\ln \left(\mathcal{C} \frac{E_{2} B_{2}\left(2 J_{2}+1\right)}{E_{1} B_{1}\left(2 J_{1}+1\right)} \frac{N_{\mathrm{p} 1}}{N_{\mathrm{p} 2}}\right)} .
$$

In a typical temperature measurement, one measures the fluorescence signal $N_{\mathrm{p}}$ and laser pulse energy $E$ for each rotational level. Then, using calculated values for $F_{J^{\prime \prime}}$ and $B_{J^{\prime} J^{\prime \prime}}$, the temperature can be obtained. This assumes that the value of $\mathcal{C}$ is known or has been determined empirically. When more than two lines are used a Boltzmann plot of

$$
\ln \left(\frac{N_{\mathrm{p}}}{E B_{J^{\prime} J^{\prime \prime}}\left(2 J^{\prime \prime}+1\right)}\right)
$$

versus $F_{J^{\prime \prime}}$ gives a straight line with slope $-1 /\left(k T_{\text {rot }}\right) . \quad$ The $y$-intercept is $\ln \left(C^{\star}\right)$ where
$C^{\star}=N_{\mathrm{T}} G \Phi \Omega \eta \ell \exp \left(-G_{v^{\prime \prime}} / k T_{\mathrm{vib}}\right) / Z_{\text {total }}$ is assumed to be a constant independent of $J$.

Vibrational temperature can be measured in an analogous way. Measuring fluorescence images for two or more transitions with different $v^{\prime \prime}$ and plotting

$$
\ln \left(\frac{N_{\mathrm{p}}}{E B_{J^{\prime} J^{\prime \prime}}\left(2 J^{\prime \prime}+1\right) \exp \left(-F_{J^{\prime \prime}} / k T_{\mathrm{rot}}\right)}\right)
$$

versus $G_{v^{\prime \prime}}$ gives a straight line with slope $-1 /\left(k T_{\text {vib }}\right)$. By keeping $F_{J \prime}$ as constant as possible, its influence is minor, and the vibrational temperature is therefore determined independent of the rotational temperature.

\section{Assumptions and Calibration}

The constant $\mathcal{C}$ in Eq. (10) can be written such that all the quantities related to the detection system are collected into a new constant $\mathcal{C}^{\prime}$. Hence, we have

$$
\mathcal{C}^{\prime}=\frac{\Omega_{1} \eta_{1} \ell_{1}}{\Omega_{2} \eta_{2} \ell_{2}}
$$

and

$$
\mathcal{C}=\mathcal{C}^{\prime} \frac{N_{\mathrm{T} 1} G_{1} \Phi_{1}}{N_{\mathrm{T} 2} G_{2} \Phi_{2}} .
$$

For PLIF thermometry in unsteady or turbulent flows, it is necessary to use two lasers and two cameras. The two transitions are excited in rapid sequence, thus freezing the flow motion, and ensuring that the flow conditions remain the same for both measurements (i.e., $N_{\mathrm{T} 1}=N_{\mathrm{T} 2}$ ). However, for steady or repeatable flows it suffices to use one laser and camera and assume a degree of flow reproducibility. For pulsed facilities it is ideal to make the measurements on a single shot as each tunnel run is not perfectly repeatable. In the current work the measurements were made over many tunnel runs because it was not possible due to monetary constraints to use two laser/camera systems. To reduce the influence of shot-to-shot flow variations, five runs were averaged for each transition, and the shock speed was monitored. Tunnel runs where the shock speed varied by more than $1 \%$ from the average were rejected. If a single detection system is used then $\Omega_{1} \eta_{1}=\Omega_{2} \eta_{2}$, and if we assume perfect flow reproducibility $N_{\mathrm{T} 1}=N_{\mathrm{T} 2}$, and therefore $\mathcal{C}^{\prime}=1$.

In Eq. (8), we assumed that the ratios $\Phi_{1} / \Phi_{2}$ and $G_{1} / G_{2}$ were independent of temperature. This is a good assumption if $\Phi$ and $G$ are independent of $J$ and reabsorption of fluorescence is negligible. ${ }^{15}$ For the $A^{2} \Sigma^{+}\left(v^{\prime}=0\right)$ band of $\mathrm{NO}$, the spontaneous emission rates and collisional quenching rates are independent of rotational quantum number. ${ }^{16}$ Rotational-level-dependent quenching has been observed in $\mathrm{OH} A^{2} \Sigma^{+}$for low- $J$ and temperatures in the range $300-$ $1200 \mathrm{~K}$. According to Paul et al. ${ }^{17}$ and based on computational models, it should also manifest in $\mathrm{NO}^{2} \Sigma^{+}$at $J<5$ when temperatures are less than $300 \mathrm{~K}$. $J$-dependent quenching would cause a significant systematic error in low-temperature NO thermometry if low- $J$ lines were used. Although low- $J$ lines are used in the current work, systematic errors are avoided because the temperatures measured are greater than $300 \mathrm{~K}$. Hence we have $A_{\text {eff } 1}=A_{\text {eff2 }}$ and $Q_{1}=Q_{2}$ and therefore $\Phi$ is also independent of $J$.

The overlap integral $G$ depends on the laser lineshape $L(\nu)$ and the absorption lineshape $Y(\nu)$. The absorption line shape, $Y(\nu)$, is affected by collisional broadening and shifting, ${ }^{18,19}$ Doppler broadening, and Doppler shifts due to the flow velocity. Only the Doppler broadening varies with $J$ but for transitions in the same vibrational band, $\nu_{a 1} \approx \nu_{a 2}$, and so $Y(\nu)$ can be considered independent of $J$. Provided the laser-line profile overlaps the absorption-line profile in the same way for both transitions, $G_{1}=G_{2}$. The overlap integral will vary throughout the flowfield due to variations in pressure and temperature, but will remain independent of $J$. For vibrational measurements the Doppler width increases by $8 \%$ between 225 and $245 \mathrm{~nm}$ which causes the overlap 


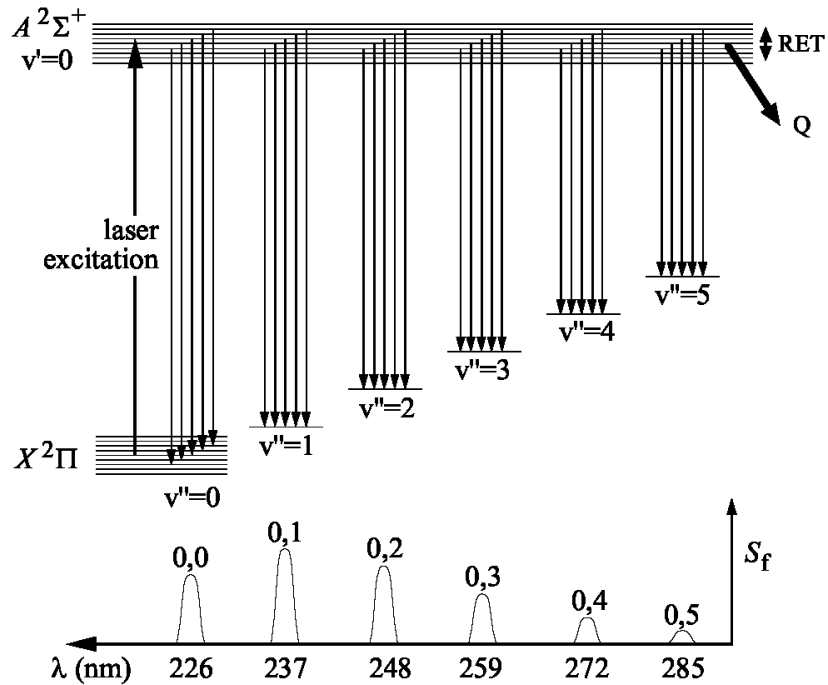

Fig. 1 The NO $A^{2} \Sigma^{+} \leftarrow X^{2} \Pi$ electronic transition. The fluorescence spectral distribution is shown at the bottom of the figure. The branching ratios were taken from Piper. ${ }^{20}$

integral to increase by $2.2 \%$. This produces measured vibrational temperatures which are systematically high by $+0.4 \%$. From the above considerations and the fact that $\mathcal{C}^{\prime}=1$, it follows that $\mathcal{C}=1$. Provided that the assumptions about the NO spectroscopy are valid, then the main concern here is that the laser is tuned to the transition in the same manner for both lines.

Ideally one would prefer to test these assumptions by calibrating the whole system at a range of known temperatures. A suitable calibration device operating at similar pressures and temperatures to the shock tunnel flow is difficult to find. A continuously running device might at least enable Boltzmann plots to be obtained where deviations from a straight line may indicate systematic errors due to the measurement system. In the current work, with no such device available, the system was tested by performing measurements in the shock-tunnel test section filled with a static $\mathrm{NO} / \mathrm{N}_{2}$ mixture at room temperature. The main difficulty this presented was due $J$-dependent attenuation of the laser beam along the $150-\mathrm{mm}$ beam path from the entrance window to the imaged region. Only when transitions with similar beam attenuation were chosen did the measured temperatures agree with the ambient temperature.

The above analysis ignores the effect of laser-mode fluctuations on the overlap integral. The radiation contains longitudinal modes and the amount of radiation in each mode changes from pulse-to-pulse. If the homogeneous absorption linewidth is much greater than the laser linewidth these fluctuations would have little impact. All the modes would fall under the absorption profile. For a dye-laser cavity of $30 \mathrm{~cm}$, the cavity-mode spacing is $0.017 \mathrm{~cm}^{-1}$ which corresponds to 11 modes excited under the $0.18 \mathrm{~cm}^{-1}$ FWHM laser profile. It should be noted that the fluctuations may be reduced by normalising the fluorescence images by LIF measurements performed simultaneously in a separate reference cell. Therefore each laser pulse can be corrected for overlap-integral fluctuations. This approach was used elsewhere ${ }^{21}$ to reduce the standard deviation in temperature measurements by a factor of 3 in comparison with energy corrections alone. In the current work the pressures are quite low and the freestream homogeneous absorption linewidth is $\sim 0.02 \mathrm{~cm}^{-1}$, only marginally larger than the mode spacing. Hence, mode fluctuations cause large variations in the measured shock-tunnel fluorescence signals. Also, the fluorescence images were normalised only by the total laser energy. From 50 tunnel runs the standard deviation of the fluorescence signal was found to be $\sim 25 \%$. Averaging 5 to 8 images for each transition helps
Table 1 The calculated freestream conditions at $285 \mathrm{~mm}$ from the nozzle throat. $I_{\text {sat }}$ was determined experimentally.

\begin{tabular}{lr}
\hline \hline Parameter & Freestream \\
\hline Temperature (K) & 396 \\
Pressure $(\mathrm{kPa})$ & 4.4 \\
Doppler FWHM $\left(\mathrm{cm}^{-1}\right)$ & 0.11 \\
Collision FWHM $\left(\mathrm{cm}^{-1}\right)$ & 0.02 \\
Collision shift $\left(\mathrm{cm}^{-1}\right)$ & -0.006 \\
Overlap integral $G(\mathrm{~cm})$ & 3.94 \\
Collisional quenching rate $Q\left(\mathrm{~s}^{-1}\right)$ & $3.8 \times 10^{6}$ \\
RET rate (s-1) & $4.0 \times 10^{8}$ \\
Fluorescence lifetime $(\mathrm{ns})$ & 114 \\
Saturation irradiance $I_{\text {sat }}\left(\mathrm{kW}^{-1} \mathrm{~cm}^{2}\right)$ & $160 \pm 22$ \\
\hline \hline
\end{tabular}

to reduce the influence of these fluctuations and leads to an estimated uncertainty in the rotational temperature of $4 \%$ (due to laser-mode fluctuations alone).

An alternative way to calibrate a LIF image is to use a known temperature somewhere in the image. In supersonic flows, this calibration point can be obtained from the stagnation point on a body or alternatively from the freestream temperature, if either is known. Another technique which measures accurately at a single point may also be used, for example, single-pulse/single-point broadband CARS thermometry. ${ }^{8}$

\section{Thermometry Strategy for Current Work}

The format for the experiments was as follows. The light from a single narrowband laser system was formed into a sheet and used to probe the shock tunnel flow. An intensified CCD camera collected the fluorescence signal at right angles to the sheet. Only one image was obtained per tunnel run. To make temperature measurements, different absorption transitions were probed on separate tunnel runs. Generally eight images were produced for each transition, and averaged. The temperature thus determined is an average of the flow conditions for that series of shock tunnel runs.

The excitation scheme employed involves excitation of $\mathrm{NO}$ through the $A^{2} \Sigma^{+} \leftarrow X^{2} \Pi\left(v^{\prime \prime}=0\right)$ band at $225 \mathrm{~nm}$ (see Fig. 1). Fluorescence is then collected from the $v^{\prime}=0 \rightarrow$ $v^{\prime \prime}=2,3$ and 4 vibrational bands by spectrally filtering the fluorescence with reflective filters. Detection at the excitation wavelength is avoided to prevent contributions to the signal from laser scatter. Furthermore, fluorescence transitions ending in the less-populated vibrational bands $\left(v^{\prime \prime}>1\right)$ are preferred to reduce the influence of fluorescence trapping, which occurs when fluorescence photons are reabsorbed by molecules between the interaction-volume and the detector. Broadband-fluorescence detection was employed to collect the fluorescence from all the rotational levels populated by collisions and not just the laser-coupled level. ${ }^{2}$ This has two advantages. First, collecting all the fluorescence gives higher fluorescence intensities. Second, most modern lasers have a high degree of polarisation which can make the fluorescence from the laser coupled level highly anisotropic. ${ }^{22}$ The levels populated by RET have reduced anisotropy due to depolarising collisions and thus collecting the broadband fluorescence significantly reduces these effects.

\section{PLIF Transition Selection}

The freestream conditions were calculated using the quasi-one-dimensional nozzle flow code STUBE as described in the section below on flow modelling. These conditions were then used to calculate the various spectroscopic quantities that influence the selection of transitions. For example, at the estimated freestream temperature and pressure, the absorption linewidths are small $\left(0.11 \mathrm{~cm}^{-1}\right)$ and therefore a 


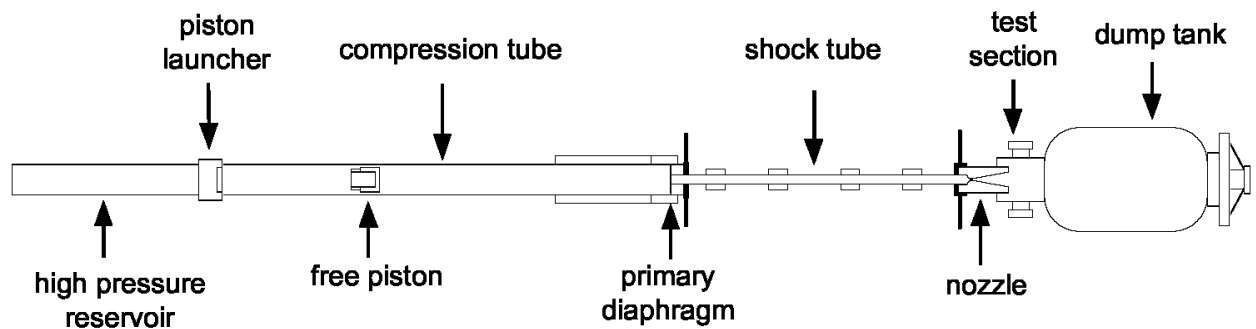

Fig. 2 The T2 free-piston shock tunnel.

Table 2 Transitions selected for thermometry.

\begin{tabular}{lllrrrr}
\hline \hline & \multicolumn{3}{c}{ Transition } & $\begin{array}{r}\text { Frequency } \\
\left(\mathrm{cm}^{-1}\right)\end{array}$ & $\begin{array}{r}F_{J} \\
\left(\mathrm{~cm}^{-1}\right)\end{array}$ & $\begin{array}{r}G_{v} \\
\left(\mathrm{~cm}^{-1}\right)\end{array}$ \\
\hline & & $v^{\prime \prime}$ & & & \\
$\mathrm{a}$ & 0 & ${ }^{\circ} P_{12}$ & 2.5 & 44069.416 & 73.58 & 948.66 \\
$\mathrm{~b}$ & 1 & $R_{2}$ & 2.5 & 42229.348 & 73.41 & 2824.76 \\
$\mathrm{c}$ & 2 & ${ }^{5} R_{21}$ & 8.5 & 40562.179 & 71.37 & 4672.68 \\
$\mathrm{~d}$ & 0 & $R_{2}$ & 23.5 & 44382.109 & 1045.85 & 948.66 \\
$\mathrm{e}$ & 1 & $R_{2}$ & 23.5 & 42516.527 & 1035.33 & 2824.76 \\
\hline \hline
\end{tabular}

Table 3 Summary of flow conditions. All values are calculated except for the shock speed and reservoir pressure which were measured. The nozzle-exit conditions are given at $285 \mathrm{~mm}$ from the nozzle throat. Uncertainties are determined by varying the input parameters to the codes.

\begin{tabular}{|c|c|c|}
\hline \multicolumn{3}{|c|}{ Nozzle-reservoir conditions (from ESTC): } \\
\hline$u_{\mathrm{s}}$ Shock speed $(\mathrm{km} / \mathrm{s})$ & 2.34 & \pm 0.02 \\
\hline$P_{0}$ Pressure $(\mathrm{MPa})$ & 27.9 & \pm 0.7 \\
\hline$T_{0}$ Temperature $(\mathrm{K})$ & 4430 & \pm 50 \\
\hline$\rho_{0}$ Density $\left(\mathrm{kg} / \mathrm{m}^{3}\right)$ & 21.2 & \pm 0.5 \\
\hline$h_{0}$ Enthalpy (MJ/kg) & 5.58 & \pm 0.08 \\
\hline \multicolumn{3}{|c|}{ Nozzle-exit conditions (from STUBE): } \\
\hline$P_{\infty}$ Static pressure (kPa) & 4.36 & \pm 0.2 \\
\hline$T_{\infty}$ Temperature $(\mathbf{K})$ & 417 & \pm 10 \\
\hline$\rho_{\infty}$ Density $\left(\mathrm{kg} / \mathrm{m}^{3}\right)$ & 0.0353 & \pm 0.08 \\
\hline$u_{\infty}$ Velocity $(\mathrm{km} / \mathrm{s})$ & 3.11 & \pm 0.02 \\
\hline$M_{\text {frozen }}$ Frozen Mach number & 7.74 & \pm 0.02 \\
\hline \multicolumn{3}{|c|}{ Vibrational freezing temperatures: } \\
\hline$T_{v}^{N_{2}}(\mathrm{~K})$ & 2150 & \\
\hline$T_{v}^{O_{2}}(\mathrm{~K})$ & 1380 & \\
\hline$T_{v}^{N O}(\mathbf{K})$ & 670 & \\
\hline
\end{tabular}

large number of lines were well isolated. A summary of the freestream conditions appears in Table 1.

Lines were chosen on the basis of: a large value of separation in ground state energies to maximise temperature sensitivity; isolation from nearby lines; minimal attenuation of the laser beam; minimal saturation of the transition; and a signal strength above the minimum detectable signal level of the detector. The transitions selected are shown in Table 2. All the transitions have very low beam attenuation and are well isolated. Transitions with similar $F_{J}$ values were chosen so that vibrational temperature measurements could also be made.

The saturation irradiance $I_{\text {sat }}$ is defined as the laser irradiance $I$ at which the LIF signal $S_{\text {sat }}$ has reduced to half the value it would have in the absence of saturation, or its linear value $S_{\text {linear. }}{ }^{3}$ This can be written as $S_{\text {sat }}=S_{\text {linear }}\left(1 /\left(1+I / I_{\text {sat }}\right)\right)$. The influence of saturation is minimized by choosing transitions in weak branches and by varying the laser energy to achieve the same level of saturation on each transition $\left(I / I_{\text {sat }} \sim 2 \%\right)$. Many lines in the $\mathrm{NO}$ spectrum consist of overlapping line pairs, where two transitions originate from the same lower level but terminate on closely spaced upper levels. The separation of these upper levels is determined by the spin-rotation splitting constant of the $A^{2} \Sigma^{+}$state. These line-pairs can be used for thermometry if saturation is negligible, as in Ref. 10, but in the current work they were avoided because negligible saturation could not be assured.

\section{Flow Modelling and the Facility}

The nozzle-reservoir conditions were calculated using the one-dimensional Equilibrium Shock Tube Code (ESTC). ${ }^{23}$ ESTC uses the initial shock tube fill pressure and temperature, test gas composition, measured shock speed, and measured reservoir pressure to determine the reservoir temperature. This temperature is then used in the quasione-dimensional inviscid nozzle-flow code STUBE. ${ }^{24}$ Onedimensional codes are very useful as engineering tools since they provide reasonably accurate solutions in only a few seconds.

STUBE was modified to include a simple vibrationalfreezing model. The vibrational-relaxation time was calculated using the theory of Landau and Teller and the constants from Ref. 25 . Using this relaxation time it was possible to determine the point during the nozzle expansion at which the vibrational temperature freezes. A sudden freezing criterion is imposed and the vibrational temperature held constant for the remainder of the expansion. The model accounts only for vibrational-translation (V$\mathrm{T}$ ) energy transfers and vibrational-vibrational (V-V) energy transfers are ignored. Due to limited availability of relaxation-rate data, the Landau-Teller rate constant for NO-N $N_{2}$ collisions was assumed to be the same as that for NO-NO collisions. However, the relaxation rate for NO-NO collisions is greater than that for the $\mathrm{NO}-\mathrm{N}_{2}$ collisions. ${ }^{26}$ The model therefore overestimates the relaxation rate of NO and thus underpredicts the NO vibrational freezing temperature. This has little effect on the other calculated nozzle-exit parameters since NO is a minority species in the current experiment. It does, however, provide a point of comparison with the experimentally measured NO vibrational temperature.

Figure 2 shows a schematic of the T2 shock tunnel. ${ }^{1}$ A $1.20 \mathrm{-kg}$ piston is free to move inside the $3.1-\mathrm{m}$ compression tube which has an internal diameter of $76 \mathrm{~mm}$. The shock tube is $1.98 \mathrm{~m}$ in length and has an internal diameter of $21 \mathrm{~mm}$. Attached to the end of the shock tube is a converging-diverging nozzle which exits into a test section which has optical access through 4 window ports. For the current experiments, the primary diaphragm was made from 0.74-mm-thick mild steel which has a burst pressure of $46.9 \mathrm{MPa}$. The secondary diaphragm was made from 0.025-mm-thick mylar. The present study employed a $7.5^{\circ}$ half-angle axisymmetric conical nozzle. The throat of the nozzle is $6.35 \mathrm{~mm}$ in diameter, the axial length of the ex- 


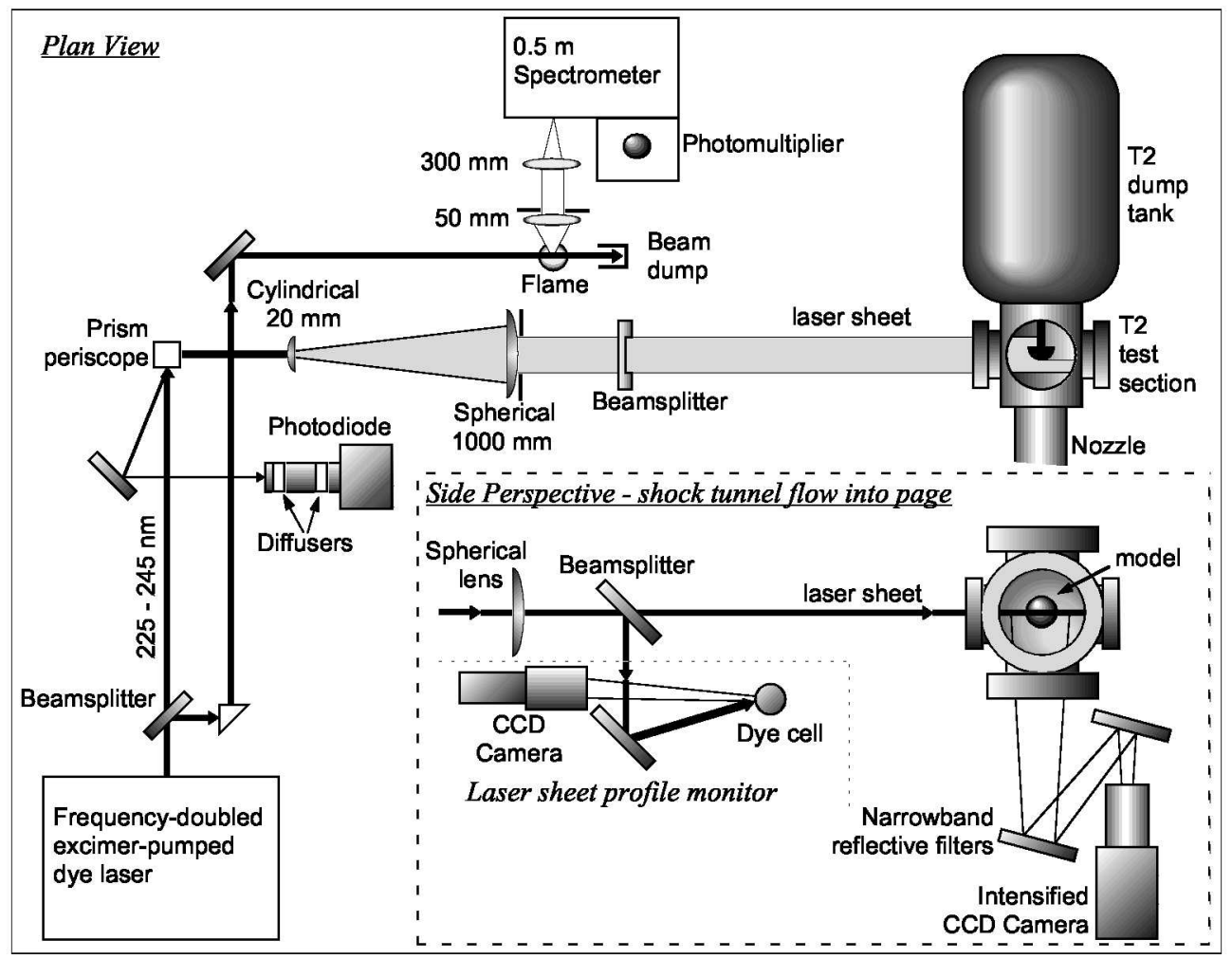

Fig. 3 Experimental apparatus for LIF imaging experiments.

panding section is $255 \mathrm{~mm}$ and the nozzle-exit diameter is $73.6 \mathrm{~mm}$. A pitot-pressure survey was conducted at the nozzle exit to estimate the size of the inviscid core. A reduced nozzle angle of $7.0^{\circ}$ was used with STUBE to account for the effect of the boundary layers on the nozzle walls.

The tunnel was operated with a helium/argon driver (81.3 kPa He, $37.3 \mathrm{kPa} \mathrm{Ar}$ ) to obtain tuned-piston operation. $^{27,28}$ The shock tube was filled with $100 \mathrm{kPa}$ of a mixture of $1.1 \% \mathrm{O}_{2}$ in $\mathrm{N}_{2}$ at $293 \mathrm{~K}$. The piston is driven by air from the high-pressure reservoir which is filled to 4.34 MPa just prior to the shot. When the piston is released it is propelled down the compression tube, compressing the driver gas and causing the diaphragm to rupture. A shock wave propagates through the test gas in the shock tube and its speed is measured by three timing transducers along its length. The transducers are placed at 1281, 316 and $11 \mathrm{~mm}$ from the nozzle inlet. The speed computed based on the shock transit time between the first two transducers was $2.49 \mathrm{~km} / \mathrm{s}$ and between the last two it was attenuated to $2.34 \mathrm{~km} / \mathrm{s}$ due to viscous effects. The shock speed is expected to decrease rapidly near the start of the shock tube and be relatively constant by the time it gets to the nozzle inlet. Hence, the value of $2.34 \mathrm{~km} / \mathrm{s}$ was used in calculating the nozzle-reservoir conditions. The shock wave reflects at the shock tube end producing a high pressure and temperature reservoir of test gas. The measured nozzle-reservoir pressure was $27.9 \mathrm{MPa}$. From these values the reservoir temperature was calculated using ESTC to be $4430 \mathrm{~K}$, and the total enthalpy was $5.8 \mathrm{MJ} / \mathrm{kg}$. The conditions calculated using STUBE at $285 \mathrm{~mm}$ from the nozzle throat are shown in Table 3.

\section{Experiment}

The experimental arrangement is shown in Fig. 3. The laser source is a frequency-doubled excimer-pumped dye laser producing up to $6 \mathrm{~mJ}$ of radiation at $225 \mathrm{~nm}$, with a linewidth of $0.18 \mathrm{~cm}^{-1}$. The pump laser was a XeCl excimer laser (Lambda Physik, EMG 150 ETS) operated as an unstable resonator, and produced approximately $250 \mathrm{~mJ}$ at $308 \mathrm{~nm}$. The dye laser (Lambda Physik, Scanmate II) was operated with different Coumarin laser dyes (Lambdachrome LC4500, 4700, 4800) to produce $40 \mathrm{~mJ}$ of tunable narrowband radiation between 450 and $490 \mathrm{~nm}$. This was then frequency doubled in a BBO I crystal to produce about $6 \mathrm{~mJ}$ of UV light between 225 and $245 \mathrm{~nm}$. Approximately $10 \%$ of the laser radiation was split off with a beamsplitter (uncoated fused silica) and directed through a small turbulent flame to perform LIF excitation scans. The NO is produced by entrainment of the air in the flame. The LIF was collected at right angles to the beam and then imaged onto a $0.5-\mathrm{m}$ spectrometer. The spectrometer was operated as a 9-nm bandpass filter which allowed detection of LIF from the NO $(0,1)$ or $(0,2)$ vibrational bands while blocking the very strong $\mathrm{OH}$ fluorescence at $308 \mathrm{~nm}$. The flame was chosen (rather than a reference cell containing NO) so that excitation scans of the $v^{\prime \prime}=1$ and 2 vibrational bands of $\mathrm{NO}$ could also be performed.

The remaining laser radiation passes through a periscope to bring the beam to the same level as the shock-tunnel test section. An $80-\mathrm{mm}$ wide sheet was produced using a 20 mm-focal-length cylindrical lens and a large spherical lens (1000-mm focal length, 100-mm diameter). The variation in energy across the laser sheet was measured simultaneously by use of a dye cell (100-mm length) and a standard CCD camera (Pulnix TM-760). ${ }^{29}$ This energy distribution measurement was used to correct the PLIF image obtained on the same laser pulse. The laser sheet was focussed in front of the test section so that the sheet thickness at the test section was $0.8 \mathrm{~mm}$. This reduced the possibility of saturation. The total beam energy was monitored with a back reflection from a prism using a UV-sensitive photodiode. The cylindrical lens overexpands the beam considerably so that the total sheet energy was reduced to only $\sim 60 \mu \mathrm{J}$. This corresponds to an irradiance of $3.7 \mathrm{~kW} / \mathrm{cm}^{2}$, where we have used a sheet area of $0.64 \mathrm{~cm}^{2}$ and assumed a laser pulse duration of $25 \mathrm{~ns}$.

The saturation irradiance was measured using a static $\mathrm{NO} / \mathrm{N}_{2}$ mixture in the shock tunnel test section, as described in the section on calibration. Making allowance for the change in conditions between this static measurement 


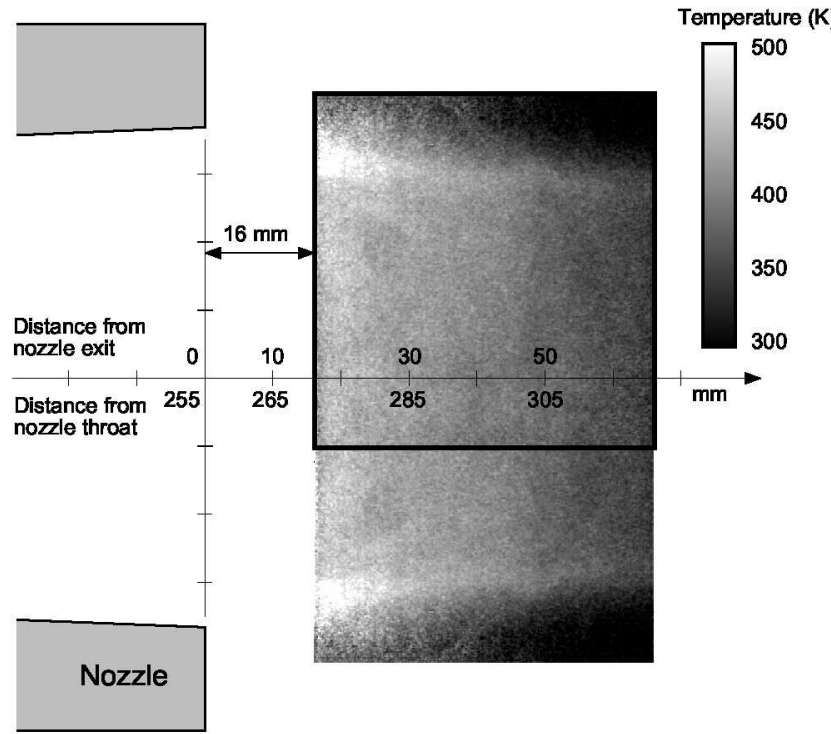

Fig. 4 Schematic of nozzle exit. The box indicates the region imaged by the camera. The imaged region is $50 \times 52 \mathrm{~mm}$. The image shown is a rotational temperature map which has been reflected about the flow centreline.

and the freestream conditions, the estimated saturation irradiance for the freestream is $160 \mathrm{~kW} / \mathrm{cm}^{2}$. Therefore the laser irradiance used for the experiment was only $2 \%$ or the saturation irradiance $\left(I / I_{\text {sat }} \sim 0.02\right)$.

The fluorescence from the test section was collected at right angles to the sheet and reflected from two mirrors and into the intensified CCD camera. The mirrors are reflective filters, designed to maximise the fluorescence signal and minimize flow luminosity. The detector used for these experiments was an in-house assembled intensified CCD camera. $^{30}$ The CCD camera (EEV Photon, P/N $\mathrm{P} 45580 / \mathrm{V} 5.3 / \mathrm{PHO}$ ) is an 8-bit asynchronous device which is triggered separately from the intensifier. The fluorescence lifetime in the freestream is $\sim 114 \mathrm{~ns}$ and so an intensifier gate of $650 \mathrm{~ns}$ was used. This allows greater than $99 \%$ of the signal to be collected and about $40 \mathrm{~ns}$ between the start of the gate and the laser pulse to account for time jitter in the triggering system. Several 'laser off' shots were also performed to verify that there was no flow luminosity.

\section{Procedure}

To ensure an accurate measurement of the laser energy used in the experiment, it was necessary to ensure that the test-section windows were cleaned prior to each tunnel run. The tunnel was then reloaded and pumped to less than $25 \mathrm{~Pa}$. A LIF excitation scan was then performed in the flame which ensured that the laser was tuned to the correct NO transition. The laser was run at $1 \mathrm{~Hz}$ while the tunnel was being filled. Immediately before firing $(<2 s)$, the tunnel operator stopped the laser via a remote switch next to the firing valve. After the firing valve was opened, the nozzle-reservoir pressure transducer detected the shock arrival at the end of the shock tube and the laser was fired $350 \mu$ s later. This delay was chosen to coincide with flow establishment in the nozzle and prior to the expected arrival time of driver gas. The intensified camera and the sheet monitoring camera were also triggered from the nozzlereservoir pressure transducer after a suitable delay.

\section{Results and Discussion}

The imaged region is defined by the box in Fig. 4 which shows a rotational temperature map at the nozzle exit. The flow is from left to right. For these experiments, only half of the freestream was imaged, and this $50 \times 52-\mathrm{mm}$ region incorporates the flow centreline. The camera actually views a $55-\mathrm{mm}$ wide region, but the laser sheet is reduced to $50 \mathrm{~mm}$

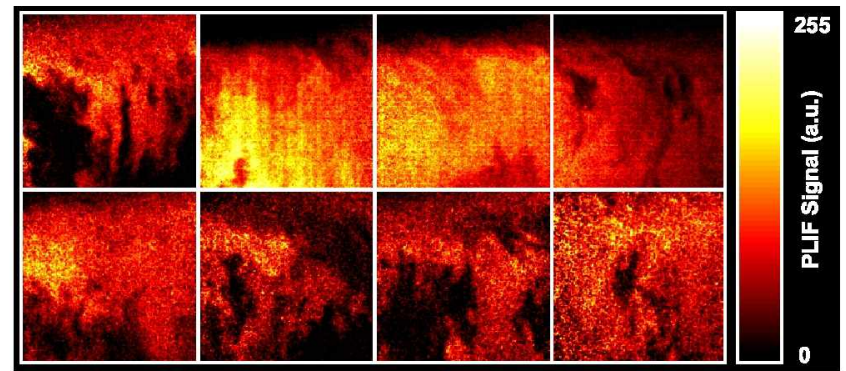

Fig. 5 A collection of LIF images showing flow nonuniformities. The position of each image relative to the nozzle is shown on Fig 4.

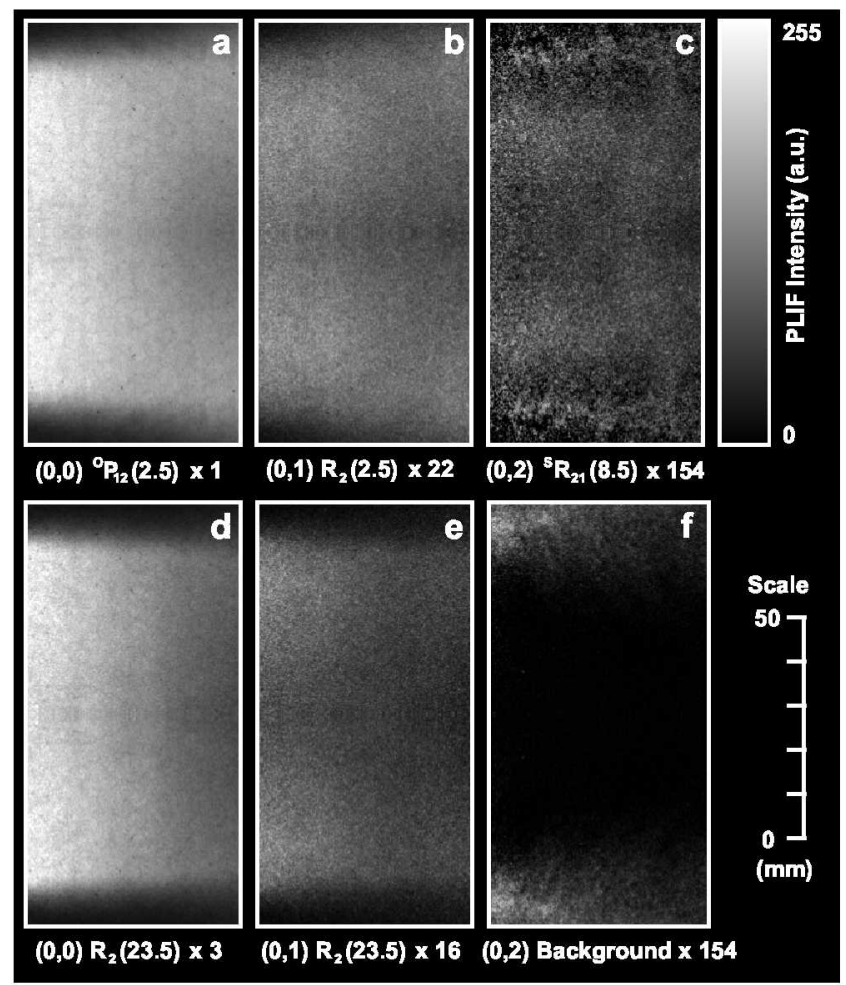

Fig. 6 Averaged LIF images of the freestream. The flow is from left to right. Images (a), (b) and (c) are for the low- $J$ lines and show the same characteristic shape. Images (d) and (e) are for the high- $J$ lines. Image (f) is the signal produced when the laser is detuned from a transition at $245 \mathrm{~nm}$. Image (c) includes the correction for the non-resonant background (f).

so that the edges of the sheet observed in the LIF image can be correlated with the sheet-energy correction image. The imaged region starts at $16 \pm 2 \mathrm{~mm}$ from the nozzle exit and includes a portion of the centreline as well as the edge of the core flow. Here, allowance has been made for the recoil of the shock tunnel when it is fired, which causes the nozzle to move $8 \pm 1 \mathrm{~mm}$ further away from the imaged region. Note that the bottom part of the image outside the box is simply the reflection of the top portion of the image.

For a uniform freestream flow, a uniform Hluorescence signal is expected. However, during the course of the experiments, several flow non-uniformities were observed (see Fig. 5), particularly along the centreline of the flow. Regions of no LIF signal were observed in approximately one third of the 68 tunnel runs performed. They have the appearance of small turbulent clouds of gas.

Several explanations exist for these nonuniformities. First, these dark regions may be explained as pockets of driver gas that have arrived prematurely, perhaps due to instabilities at the contact surface or jetting of driver gas into the nozzle-reservoir region via shock wave/boundary 


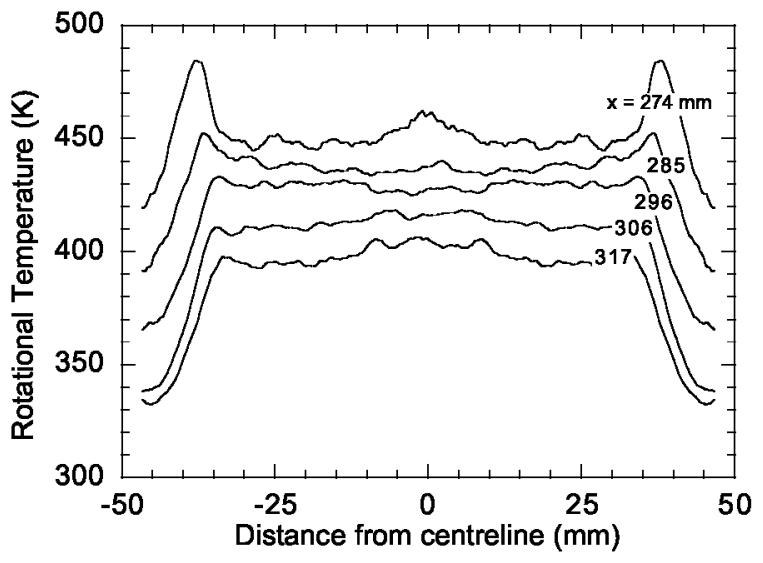

Fig. 7 Cross sections through the rotational temperature map perpendicular to the flow direction.

layer interaction. ${ }^{31,32}$ The driver gas has no NO in it and hence produces no LIF signal. Second, because the NO used in the current experiment is generated in the shockreflection process, there exists a boundary layer of test gas on the shock-tube walls without NO. This boundary layer may mix with the test gas in a similar method to the shock wave/boundary layer interaction described above. Third, the lack of signal may be due to particulate matter obstructing the fluorescence between the plane of the laser sheet and the exit window. It is known that the nozzle-boundary layer entrains particulate matter from the nozzle walls since this has been observed by Mie scatter when detecting at the laser wavelength (see Fig. 6(f)). However, if this was the cause then one would also observe sudden drop outs in the laser beam which would cause streaks in the fluorescence signal. These are never observed so the it is more likely one of the first two scenarios is correct. Attempts to seed the driver gas with NO produced negative results which are inconclusive.

It is interesting to note that very little mixing has occurred, as indicated by the high contrast of some of the 'dark-gas' pockets. At later test times $(>350 \mu \mathrm{s})$, LIF images display a gradual uniform decrease in intensity which may be due to either a drop in the nozzle-reservoir pressure and hence nozzle-exit density, or an increased proportion of driver gas uniformly mixed with the test gas, or both.

Individual images showing flow non-uniformities were rejected from the temperature analysis. Each image obtained from a single tunnel run was corrected for camera background offset, absolute laser energy, and the variation of the laser energy across the image. The corrected images for each transition were then averaged, typically 8 images per transition. These averaged images are shown Fig. 6. Because of the limited dynamic range of the camera and the significant variation in fluorescence signal intensities, especially from different vibrational bands, the camera intensifier gain was varied for different transitions. The intensifier gain control was therefore calibrated at the same time that linearity tests were performed on the intensifier. This explains the intensity-scaling factors at the bottom right of each image in Fig. 6. For the six images (a-f) in Fig. 6 the gains were $6.5,7.2,7.5,6.8,7.2$ and 7.5 , respectively. Images taken at higher gains, such as image (c), show significant shot noise despite averaging. The reflective filters efficiently block the laser wavelength when excitation is performed in the $(0,0)$ and $(0,1)$ bands. However, elastically-scattered laser light is not filtered out by the detection system for excitation of the $(0,2)$ band near $245 \mathrm{~nm}$. To account for this, several runs were performed with the laser at $245 \mathrm{~nm}$ but detuned from any NO transitions. The average of 6 such images is shown in Fig. 6(f). The signal occurs predominantly at the edge of the core flow and is most likely due to Mie scattering from particulate matter that has been entrained in the nozzle-wall boundary layer. There is no signal in the central

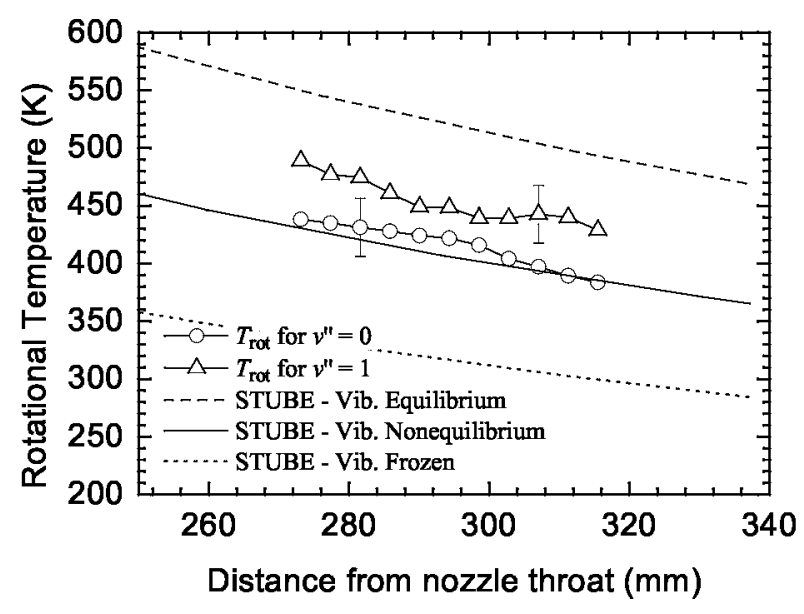

Fig. 8 Rotational temperatures along the centreline of the flow compared with STUBE calculations.

core of the flow indicating it is relatively clean of particulate matter. Image (c) is a corrected LIF image obtained by subtracting image (f) to account for the elastically-scattered laser light.

A rotational temperature map was calculated by taking the ratio of images (a) and (d) in Fig. 6 and using Eq. 10. The temperature map produced is shown in Fig. 4. However, a more quantitative assessment can be made by examining the vertical cross sections given in Fig. 7. Each cross section was made by averaging a 50-pixel-wide vertical slice through the image. The pixel density was 9.2 pixels $/ \mathrm{mm}$, and so this corresponds to approximately a $5-\mathrm{mm}$ wide slice. The data was further smoothed by running a 20-pixel-wide $(2 \mathrm{~mm})$ integration window through the data. The cross sections are at $10-\mathrm{mm}$ intervals and clearly show the decrease in temperature with distance from the nozzle exit. Note that the data shown in Fig. 7 greater than $+10 \mathrm{~mm}$ from the centreline is a reflection of the data from below $-10 \mathrm{~mm}$. The first slice, corresponding to $274-\mathrm{mm}$ from the nozzle throat, shows a marked temperature rise at the edge of the core flow. This temperature rise is caused by viscous dissipation ${ }^{33}$ in the nozzle-wall boundary layer, and on closer examination of the image in Fig. 4, can be seen to agree very well with the position of the nozzle wall. There is a small temperature rise on the centreline which may be caused to some upstream disturbance. Slices taken further downstream show a more uniform temperature profile. A second temperature map was made from transitions in the $(0,1)$ band (images (b) and (e) of Fig. 6) and showed similar characteristics, however the signal-to-noise ratio was significantly poorer.

Figure 8 shows streamwise temperature measurements compared with STUBE calculations. Each experimental data point was obtained by averaging over a $2 \times 10 \mathrm{~mm}$ region at several points along the flow centreline. The two limiting cases for vibrational relaxation are shown to indicate the confidence interval for the calculations. The upper curve represents the case when the vibrational temperature is equilibrium with the kinetic temperature while the lower curve indicates the extremely unlikely case where the vibrational temperature is frozen at the nozzle-reservoir value. The solid curve represents the case obtained by letting the $\mathrm{N}_{2}$ vibrational temperature freeze at $2150 \mathrm{~K}$ during the nozzle expansion, as explained above. Good agreement with STUBE is obtained with the temperature from the $v^{\prime \prime}=0$ level but the temperature from the $v^{\prime \prime}=1$ level is high by $30 \mathrm{~K}$.

\section{Temperature Uncertainties}

The major uncertainty in the freestream measurement is due to fluctuations in the laser spectral profile. More precisely, it is due to the fluctuation in the distribution of 


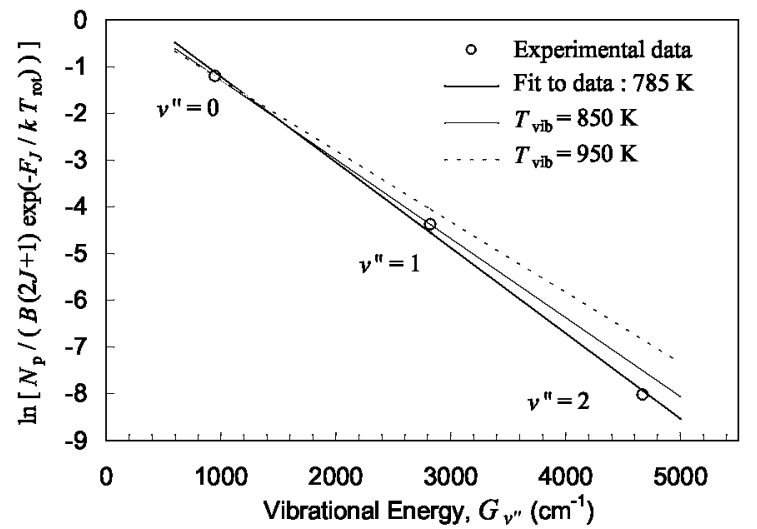

Fig. 9 Boltzmann plot for vibrational thermometry.

energy amongst the cavity modes of the laser. The uncertainty in a single image due to laser-mode fluctuations was determined by considering the data from all tunnel runs to be $25 \%$. Averaging 8 images gives an uncertainty of $9.4 \%$ for each averaged image in Fig. 6. For two-line thermometry, we use the ratio of two such images and therefore the uncertainty in the ratio is $\sqrt{2}$ greater. The uncertainty in the temperature $\delta T$ is related to the signal ratio $R$ according to

$$
\frac{\delta T}{T}=\frac{k T}{\Delta E} \frac{\delta R}{R},
$$

where $\Delta E$ is the difference between the energy levels $(\Delta E=$ $F_{J 1}-F_{J 2}$ ). For the freestream rotational-temperature measurements $\Delta E=972 \mathrm{~cm}^{-1}, T \approx 450 \mathrm{~K}$ and $\Delta E / k T \approx 3.25$, and therefore the uncertainty in the rotational temperature due to laser-mode fluctuations is only $4 \%$. Other sources of random error include the laser-energy measurements, camera gain and gate repeatability, time jitter in the laser system, laser-tuning inaccuracies and flow repeatability. All of these uncertainties are negligible in comparison with the laser-mode fluctuations.

Systematic uncertainties have been reduced or avoided by careful experiment design and transition selection. We estimate that beam attenuation, signal interference and saturation produced a combined error of less than $1 \mathrm{~K}$. It is possible that transition overlaps or other interferences may occur that are not predicted by the calculations, but these can only be discovered by temperature verification experiments. Another systematic error source is due to nonlinearities in the intensified camera system. This a possible explanation for the different rotational temperatures derived from the $v^{\prime \prime}=0$ and $v^{\prime \prime}=1$ bands. The linearity of the detector was tested by performing LIF in a static $\mathrm{N}_{2} / \mathrm{NO}$ mixture and plotting the integrated LIF intensity against laser energy. However, the test was ineffective at gains above 7.0 because shot noise caused a large number of the pixels to saturate (exceed the 8-bit dynamic range of 255 counts). The test could only indicate the camera was linear up to 100 counts. Because the signal from the low- $J$ line used for the $v^{\prime \prime}=1$ rotational temperature measurement is stronger than the high- $J$ line, it is more likely to suffer from saturation effects. A reduced low- $J$ signal would produce a higher rotational temperature as observed. The signals obtained from the $v^{\prime \prime}=0$ band were measured at lower gains where the camera was verified to be linear up to 220 counts. To get agreement between the two measured temperatures the signal from image (b) would need to be $20 \%$ greater.

\section{Vibrational Temperatures}

Freestream vibrational temperatures were also determined from the LIF images shown in Fig. 6. Using the signals from the $v^{\prime \prime}=0,1$ and 2 low- $J$ transitions (a), (b) and (c) and plotting the expression in Eq. 12 versus $G_{v^{\prime \prime}}$ gives

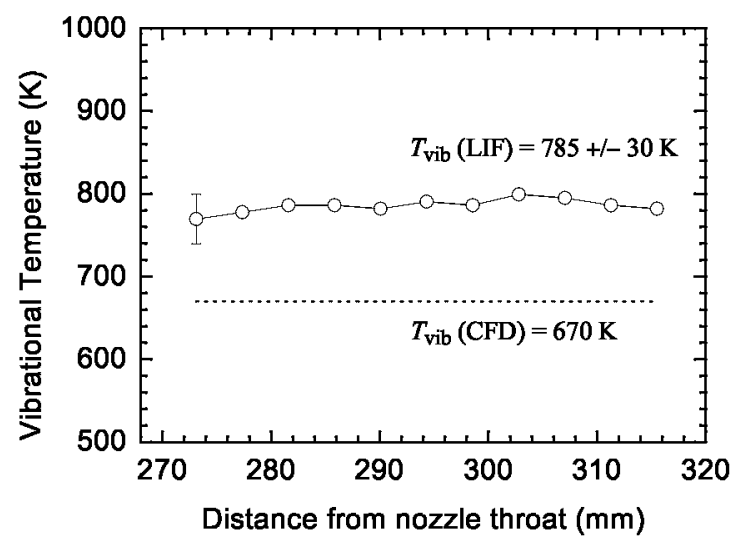

Fig. 10 Vibrational temperatures along the centreline of the flow.

a Boltzmann plot, as shown in Fig. 9. From the slope of this plot we can get the vibrational temperature. A similar approach is taken to that of the rotational measurements to reduce shot-noise uncertainties by averaging the temperature over a $2 \times 10 \mathrm{~mm}$ region. Figure 10 shows the vibrational temperatures at various distances from the nozzle exit along the flow centreline. The temperature error bars are due to laser-mode fluctuations. It is observed from Fig. 10 that the measured vibrational temperature is significantly higher than that predicted from the computational model. The temperature is constant with distance from the nozzle exit indicating that it is frozen. The measured temperature is $785 \pm 30 \mathrm{~K}$ compared with the value from the vibrational relaxation model of $670 \mathrm{~K}$. The underprediction of the vibrational freezing temperature was expected due to the lack of vibrational relaxation data.

The estimated systematic uncertainties for the vibrational temperature measurements due to beam attenuation and signal interference from nearby lines are $\delta T / T \approx+0.7 \%$ and $\approx+0.3 \%$, respectively. Uncertainties due to saturation are negligible because the laser energy was varied to achieve the same amount of saturation for each transition. The Doppler width decreases by $8.0 \%$ between 225 and $245 \mathrm{~nm}$, which causes the overlap integral to increase by $2.2 \%$. This produces measured temperatures that are systematically high by $+0.4 \%$. Combining these uncertainties, the estimated systematic error for the vibrational measurements is $+0.85 \%$, or $+7 \mathrm{~K}$.

However, it is also believed that camera nonlinearities have contributed to lowering the measured vibrational temperature in a similar way to that described for the rotational temperature measurement. From the Boltzmann plot in Fig. 9, it can be seen that the signal intensity from the $v^{\prime \prime}=2$ level is $\sim 35 \%$ less than that predicted by a straight line fit from the $v^{\prime \prime}=0$ and 1 signals. Since this signal was acquired at the highest intensifier gain it is most affected by camera saturation. A lower $v^{\prime \prime}=2$ signal would lead to a lower observed temperature. In fact if we use only the first two vibrational levels $\left(v^{\prime \prime}=0,1\right)$ we get a temperature of $\sim 850 \mathrm{~K}$. However, just as with the rotational measurements, we suspect that the $v^{\prime \prime}=1$ transitions may also be affected by camera saturation, so the actual vibrational temperature may be even higher. Increasing the $v^{\prime \prime}=1$ signal by $20 \%$ as described in the rotational measurements above leads to a temperature of $\sim 950 \mathrm{~K}$.

\section{Agreement with CFD}

To calculate the nozzle-exit conditions accurate knowledge of the nozzle-reservoir temperature is required. Here we have used the average shock speed measured between two transducers 11 and $316 \mathrm{~mm}$ from the nozzle inlet. We have assumed that this is a good estimate for the shock speed at the nozzle inlet. If the shock speed is decreas- 
ing at the linear rate along the shock tube than the shock speed at the nozzle inlet would be only $2.25 \mathrm{~km} / \mathrm{s}$, leading to an underestimate of of the nozzle-reservoir temperature by $200 \mathrm{~K}$, and nozzle-exit rotational temperature by $30 \mathrm{~K}$. Furthermore, the calculations for the nozzle-reservoir conditions (using the code $\mathrm{ESTC}^{23}$ ) make several assumptions and exclude losses due to viscous, radiation and conduction effects, driver gas contamination and shock-tunnel impurities.

If the $\mathrm{N}_{2}$ vibrational-freezing temperature could be measured, for example with broadband CARS, then it would be possible to make a reasonable estimate for the nozzlereservoir temperature. This would be one method of validating the assumptions described above used by ESTC. In related work we measured the temperature at the stagnation point on a hemisphere using PLIF. This work will be presented elsewhere, but we mention it here since a measurement of the stagnation point temperature would give a lower bound for the reservoir temperature. The vibrational temperature of $\mathrm{NO}$ at the stagnation point on a hemisphere was measured to be $4500 \pm 270 \mathrm{~K}$. Unfortunately the large uncertainty in this measurement prevents a definitive estimate for the reservoir temperature, but it demonstrates an alternative approach which may aid in validating shocktunnel CFD codes.

\section{Conclusions}

Planar laser-induced fluorescence of NO has been used to characterise the nozzle flow from a free-piston shock tunnel. Flow non-uniformities have been observed which indicate a contamination of the test gas by driver gas or test gas from the boundary layers on the shock tube walls. This is the first time to the author's knowledge that such non-uniformities have been observed. This discovery may have significant consequences for free-piston shock tunnel research, in particular in experiments involving combustion research, where a pocket of driver gas passing through a combustor could extinguish or considerably affect ignition.

A rotational temperature image was made at the nozzle exit and shows reasonable agreement with CFD calculations. Temperatures of $440 \pm 25 \mathrm{~K}$ and $460 \pm 25 \mathrm{~K}$ were measured at $285 \mathrm{~mm}$ from the nozzle throat for transitions in the $v^{\prime \prime}=0$ and 1 vibrational levels. Non-linearities in the homemade camera system are believed responsible for inconsistencies between these two measured temperatures. The first measurement was performed at lower intensifier gains where the system's linearity had been validated. The vibrational temperature of $\mathrm{NO}$ at the nozzle exit was measured to be $785 \pm 30 \mathrm{~K}$. It is believed that this temperature may actually be as high as $950 \mathrm{~K}$ and that camera non-linearities have caused a systematic error in the measurement. Nonetheless, the temperature was found to be constant with distance from the nozzle exit indicating that it is frozen.

We conclude that PLIF is a useful technique for observing flow non-uniformities and mapping flowfield distributions. More work is required to validate its accuracy, however the problem with camera non-linearities is easily solved with modern commercial camera systems. Validation of nozzle-flow codes requires improved knowledge of the nozzlereservoir conditions.

\section{Acknowledgements}

The experiments was carried out at The Australian National University. The authors would like to thank $\mathrm{Mr}$ P. Walsh for his expert technical assistance and Dr S. O'Byrne for the many hours of insightful discussions.

\section{References}

${ }^{1}$ Stalker, R. J., "A Study of the Free-Piston Shock Tunnel," AIAA Journal, Vol. 5, No. 12, 1967, pp. 2160-2165.

${ }^{2}$ Seitzman, J. M. and Hanson, R. K., "Planar Fluorescence Imaging in Gases," Instrumentation for Flows with Combustion, Academic Press Ltd, 1993
${ }^{3}$ Eckbreth, A. C., Laser Diagnostics for Combustion. Temperature and Species, Gordon and Breach Publishers, 2nd ed., 1996.

${ }^{4}$ Allen, M. G., McManus, K. R., and Sonnenfroh, D. M., "PLIF imaging in spray flame combusters at elevated pressure," $A I A A P a$ per 95-0172, 1995 .

${ }^{5}$ Quagliaroli, T. M., Laufer, G. Hollo, S. D., Krauss, R. Whithurst III, R., and McDaniel, Jr., J., "Planar KrF Laser-Induced OH Fluorescence Imaging in a Supersonic Combustion Tunnel," Journal of Propulsion and Power, Vol. 10, 1994, pp. 377-381.

${ }^{6}$ Ruyten, W. M., Smith, M. S., and Williams, W. D., "On The Role of Laser Absorption in Planar Laser-Induced Fluorescence Imaging of High-Enthalpy Flows," 20th International Symposium on Shock Waves, Pasadena, CA, 1995.

${ }^{7}$ Palma, P. C., Houwing, A. F. P., and Sandeman, R. J., "Absolute Intensity Measurements of Impurity Emissions in a Shock Tunnel and Their Consequences for Laser-Induced Fluorescence Experiments," Shock Waves, Vol. 3, No. 1, 1993, pp. 49-53.

${ }^{8}$ Hahn, J. W., Park, C. W., and Park, S. N., "Broadband Coherent Anti-Stokes Raman Spectroscopy with a Modeless Dye Laser," Applied Optics, Vol. 36, No. 27, 1997, pp. 6722-6728.

${ }^{9}$ McMillin, B. K., Palmer, J. L., and Hanson, R. K., "Temporally Resolved, Two Line Fluorescence Imaging of NO Temperature in a Transverse Jet in a Supersonic Crossflow," Applied Optics, Vol. 32, 1993, pp. 7532-7545.

${ }^{10}$ Palmer, J. L., McMillin, B. K., and Hanson, R. K., "Multi-Line Fluorescence Imaging of the Rotational Temperature Field in a Shock Tunnel," Applied Physics B, Vol. 63, No. 8, 1996, pp. 167-178.

${ }^{11}$ Koch, U., Gülhan, A., Esser, B., Grisch, F., and Bouchardy, P., "Rotational and Vibrational Temperature and Density Measurements by Planar Laser Induced NO-Fluorescence Spectroscopy in a Nonequilibrium High Enthalpy Flow," AGARD Fluid Dynamics Panel Symposium, Advanced Aerodynamic Measurement Technology, CP-601, AGARD, 1997, Paper 15.

${ }^{12}$ Grisch, F., Bouchardy, P., Joly, V., Marmignon, C., Koch, U., and Gülhan, A., "Coherent Anti-Stokes Raman Scattering Measurements and Computational Modeling of Nonequilibrium Flow," AIAA Journal, Vol. 38, No. 9, 2000, pp. 1669-1675.

${ }^{13}$ Allen, M. G., Parker, T. E., Reinecke, W. G., Hartmut, H. L., Foutter, R. R., Rawlins, W. T., and Davis, S. J., "Fluorescence Imaging of $\mathrm{OH}$ and NO in a Model Scramjet Combuster," AIA A Journal, Vol. 31, 1993, pp. 505-512.

${ }^{14}$ Laurendeau, N. M. "Temperature Measurements by LightScattering Methods," Progress in Energy Combustion Science, Vol. 14, 1988, pp. 147-170.

${ }^{15}$ McMillin, B. K., Seitzman, J. M., and Hanson, R. K., "Comparison of NO and $\mathrm{OH}$ Planar LIF Temperature Measurements in SCRAMJET Model Flowfields," AIAA Journal, Vol. 32, 1994, in SCRAMJET
pP. $1945-1952$.

pp. $1945-1952$.

${ }^{16} \mathrm{McDermid}, \mathrm{I}$. S. and Laudenslager, J. B., "Radiative Lifetimes and Electronic Quenching Rate Constants for Single-Photon-Excited Rotational Levels of NO $\left(A^{2} \Sigma^{+}, v^{\prime}=0\right)$," Journal of Quantitative Spectroscopy and Radiative Transfer, Vol. 27, 1982, pp. 483-492.

${ }^{17}$ Paul, P. H., Grav, J. A., Durant, Jr., J. L., and Thoman, Jr., J. W., "Collisional Quenching Corrections for Laser-Induced Fluorescence Measurements of $\mathrm{NO}^{2} \Sigma^{+}, " A I A A$ Journal, Vol. 32, No. 8, 1994, pp. 1670-1675.

${ }^{18}$ Chang, A. Y., Di Rosa, M. D., and Hanson, R. K., "Temperature Dependence of Collision Broadening and Shift in the NO $A \leftarrow X(0,0)$ Band in the Presence of Argon and Nitrogen," Journal of Quantitative Spectroscopy and Radiative Transfer, Vol. 47, 1992, pp. $375-390$

${ }^{19} \mathrm{Di}$ Rosa, M. D. "Collision-Broadening and -Shift of NO $\gamma(0,0)$ Absorption Lines by $\mathrm{H}_{2} \mathrm{O}, \mathrm{O}_{2}$, and $\mathrm{NO}$ at $295 \mathrm{~K}$," Journal of Molecular Spectroscopy, Vol. 164,1994, pp. $97-117$

${ }^{20}$ Piper, L. G. and Cowles, L. M., "Einstein Coefficients and Transition Moment Variation for the NO $\left(A^{2} \Sigma^{+}-X^{2} \Pi\right)$ Transition," Journal of Chemical Physics, Vol. 85, No. 5, 1986, pp. 2419-2422.

${ }^{21}$ Wollenhaupt, M., Rosenhauer, M., Müller, T., Jourdan, J., Scholz, J., Hartung, S., and Beck, W. H., "NO Laser-Induced Fluorescence Studies for the Application of Single-Shot Two-Line Thermoemtry to HEG," 21th International Symposium on Shock Waves, moemtry to HEG," 21th International

${ }^{22}$ Doherty, P. M. and Crosley, D. R., "Polarisation of LaserInduced Fluorescence of $\mathrm{OH}$ in an Atmospheric Pressure Flame," Applied Optics, Vol. 23, 1982, pp. 713-721.

${ }^{23}$ McIntosh, M., "Computer Program for the Numerical Calculation of Frozen Equilibrium Conditions in Shock Tunnels," Tech. rep., Department of Physics, Faculties, Australian National University, 1968.

${ }^{24}$ Vardavas, I., "Modelling Reactive Gas Flows within Shock Tunnels," Australian Journal of Physics, Vol. 37, 1984, pp. 157-177.

${ }^{25}$ Vincenti, W. G. and Kruger, Jr., C. H., Introduction to Physical Gas Dynamics, Kreiger, Malabar, Florida, 1975.

${ }^{26}$ Park, C., Nonequilibrium Hypersonic Aerothermodynamics, John Wiley and Sons, 1st ed., 1990.

${ }^{27}$ Hornung, H. G. and Bélanger, J., "Role and Techniques of Ground Testing for Simulation of Flows Up to Orbital Speed," AIAA Paper 90-1377, 1990 
${ }^{28}$ Itoh, K., "Tuned Operation of a free-piston shock tunnel," 20th International Symposium on Shock Waves, World Scientific, 1996 , pp. $43-51$.

${ }^{29}$ Seitzman, J. M., Hanson, R. K., Barber, P. A., and Hess, C., "Application of Quantitative Two-Line OH PLIF for Temporally Resolved Planar Thermometry in Reacting Flows," Applied Optics, Vol. 33, 1994, pp. 4000-4012.

${ }^{30}$ McIntyre, T. J., "Intensified CCD Camera System," Tech. rep., Department of Physics, Faculties, Australian National University, 1995.

${ }^{31}$ Davies, L. and Wilson, J. L., "Influence of Reflected Shock and Boundary-Layer Interaction on Shock-Tube Flows," Physics of Fluids, Vol. 12, No. 6, 1969, pp. I37-I43, Supplement I.

${ }^{32}$ Stalker, R. J. and Crane, K. C. A., "Driver Gas Contamination in a High-Enthalpy Reflected Shock Tunnel," AIAA Journal, Vol. 16, No. 3, 1978, pp. 277-279.

${ }^{33}$ Anderson, Jr., J. D., Hypersonic and High Temperature Gas Dynamics, McGraw-Hill, 1989. 